\title{
FIELDING AN AMPHIBIOUS UAV: DEVELOPMENT, RESULTS, AND LESSONS LEARNED
}

\author{
Greg Pisanich, QSS Group Inc., NASA Ames Research Center, Moffett Field, CA \\ Dr. Stephen Morris, MLB, Palo Alto, CA
}

\begin{abstract}
This report summarizes the work completed on the design and flight-testing of a small, unmanned, amphibious demonstrator aircraft that flies autonomously. The aircraft named ACAT (Autonomous Cargo Amphibious Transport) is intended to be a large cargo carrying unmanned aircraft that operates from water to avoid airspace and airfield conflict issues between manned and unmanned aircraft. To demonstrate the feasibility of this concept, a demonstrator ACAT was designed, built, and flown that has a six-foot wingspan and can fly autonomously from land or water airfield.
\end{abstract}

The demonstrator was designed for a 1-hour duration and 1-mile telemetry range. A sizing code was used to design the smallest demonstrator UAV to achieve these goals. The final design was a sixfoot wingspan, twin hull configuration that distributes the cargo weight across the span, reducing the wing structural weight.

The demonstrator airframe was constructed from balsa wood, fiberglass, and plywood. A 4stroke model airplane engine powered by methanol fuel was mounted in a pylon above the wing and powers the ACAT UAV. Initial flight tests from land and water were conducted under manual radio control and confirmed the amphibious capability of the design.

Flight avionics that were developed by MLB for production UAVs were installed in the ACAT demonstrator. The flight software was also enhanced to permit autonomous takeoff and landing from water. A complete autonomous flight from a hard runway was successfully completed on July 5 , 2001 and consisted of a take-off, rectangular flight pattern, and landing under complete computer control. A completely autonomous flight that featured a water takeoff and landing was completed on October 4, 2001. This report describes these activities in detail and highlights the challenges encountered and solved during the development of the ACAT demonstrator.

\section{Project Genesis and Long Term Goals}

The ACAT project was proposed as an amphibious platform built to test advanced information and system technologies. This testbed would support multiple program objectives including: Intelligent Vehicle Health Management, Design for Safety, Intelligent Systems, and Information Technologies Base programs. The system would test a wide range of capabilities:

- Advanced fault tolerant and self diagnosing systems

- Autonomous flight control and planning

- Advanced real-time communications and monitoring systems

The testbed would be developed in phases. The first phase consisted of a small prototype to test out the basic capabilities. Based on the results of the initial flight tests, a larger phase II system was planned that would not only fulfill the basic operating capabilities, but would also meet demonstration mission requirements. The demonstration mission requirement would consist of an autonomous flight from San Francisco to Hawaii with a minimum cargo payload onboard. 
The MLB Company of Palo Alto, CA was

responsible for the design, development, and flight control systems of the UAV.

\section{Aircraft Design and Construction}

\section{Sizing Study}

Based on the demonstration requirements of a San Francisco to Hawaii flight, MLB developed an aircraft sizing code that used numerical optimization to predict the span, area, propulsion, and fuel of the smallest aircraft that could complete that mission. Assuming a 2-pound payload for communication and miscellaneous equipment, the numerical optimization results predicted an aircraft with the following performance and characteristics:

- $\quad$ Cruise speed $=60 \mathrm{mph}$ IAS

- Range $=3000$ mile (no wind)

- Duration $=50$ hours

- Climb Rate at take-off $=350 \mathrm{fpm}$

- $\quad \operatorname{Span}=12 \mathrm{ft}$

- Area $=12 \mathrm{ft}^{2}$

- Gross Take Off Weight $=87 \mathrm{lb}$

- $\quad$ Fuel $=5$ gallons

- $\quad$ Power installed $=5 \mathrm{HP}$

- [Assumed SFC=0.4 lbfuel/(HP-hour)]

A smaller version of this aircraft was designed as the initial ACAT test aircraft. This prototype would be used to demonstrate the amphibious flight operations and test the autonomous flight control system. Its specifications were:

- $\quad$ Cruise speed $=35$ mph IAS

- $\quad$ Range $=35$ miles (no wind)

- Duration $=1$ hour

- Climb Rate at takeoff $=350 \mathrm{fpm}$

- $\quad \mathrm{Span}=6 \mathrm{ft}$

- $A r e a=6 \mathrm{ft}^{2}$

- Gross Take off Weight $=8 \mathrm{lb}$

This included a $1 \mathrm{lb}$ flight avionics weight and standard 4-stroke model airplane engine technology for propulsion.

\section{ACAT Demonstrator Configuration Design}

The numerical optimization software that was used sizes the wing and propulsion system, but does not specify the configuration. A twin-hull design was chosen to span-load the wing for reduced structural weight and for improved water handling. The engine is mounted above the wing in a pylon for maximum clearance from the water. The wing sweep and airfoil shapes of a full-size ACAT will depend on the desired cruise Mach number. The prototype design used a zero sweep wing and a Clark-Y subsonic airfoil. A detailed structural design of the aircraft was performed assuming a hull shape based on information in NACA report TN-2481 for hulls with improved planing stability. Full size plans were drawn and the construction of the airframe was initiated.

\section{ACAT Demonstrator Construction}

The ACAT airframe is built primarily from balsa and plywood with fiberglass reinforcing, epoxy paint, and Mylar covering. The conventional flight controls (aileron, dual rudders, elevator, and throttle) are operated by model airplane servos.

Figure 1 shows a series of photographs summarizing the major steps in the construction of the airframe.

A 2-stroke 0.40 cubic inch engine was initially installed in the ACAT (this was later changed to a 0.70 cubic inch 4 -stroke engine for increased power and better fuel economy). The aircraft was filled with lead ballast to mimic the avionics payload that would be added later. A removable set of coilsprung landing gears was fitted to each hull for operation from hard runways. The aircraft weighed 12 pounds with fuel at this stage.

\section{Radio Control Flight Tests}

For the first flight tests, the ACAT was flown from a hard runway using the smaller 0.4 cubic inch engine. These tests showed a slight tail-heavy condition and a need for coupling between the ailerons and rudders to eliminate adverse yaw while rolling at low speeds. The stall characteristics were docile. 


\section{Amphibious UAV Construction Sequence}

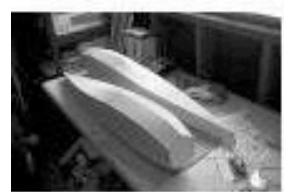

Hulls planked

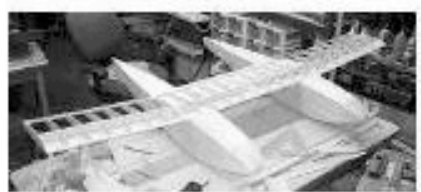

Wing framed

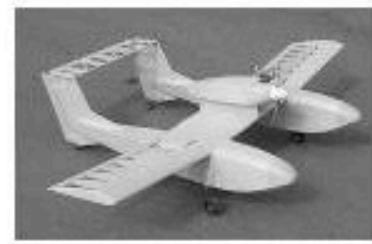

Ready for finish

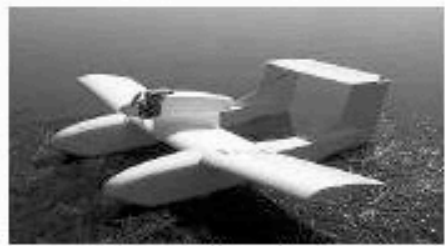

Floatation test

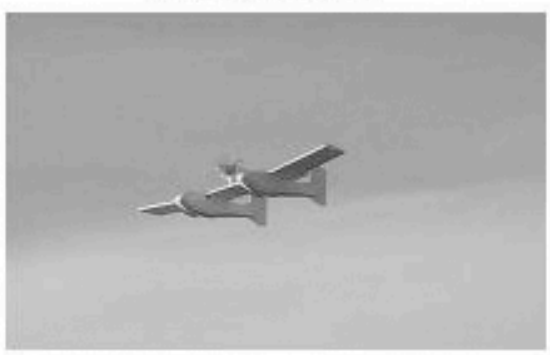

Water flight test

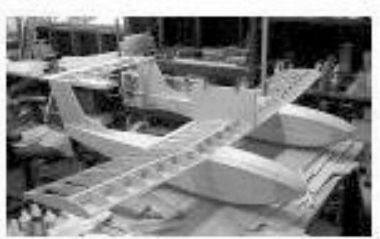

Tail framed

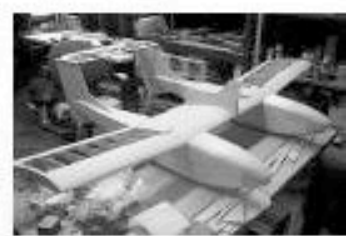

Wing sheeted

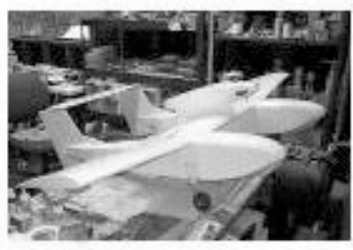

Ready to fly

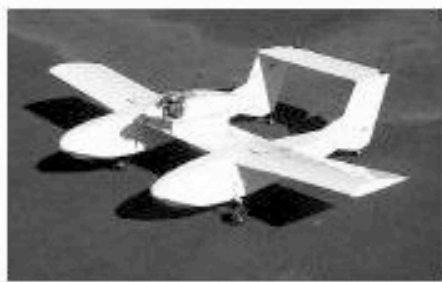

Taxi test

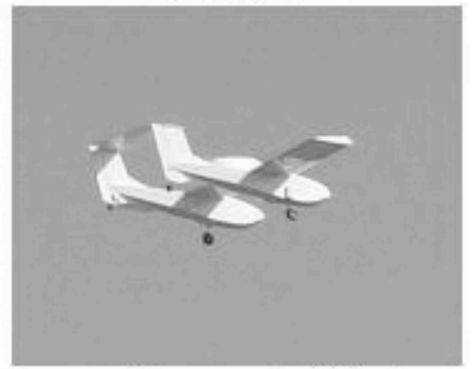

Runway flight test

Figure 1. Amphibious UAV Construction Sequence.

The pylon mounted engine produces some coupling between the thrust and pitch moment which must be compensated for as the throttle is changed. Overall, the flights from land were very successful.

The landing gear was removed and floatation tests were conducted to verify that the aircraft had a proper water line and no hull leaks. Initial attempts at water take-off showed the spray from the hulls was hitting the propeller such that the engine could not develop enough RPM to achieve planing speed. Spray fences were added to the front sides of the hulls and a smaller diameter propeller was installed with greater pitch. With these changes, the UAV was able to reach planing speed with a 50 foot take- off run. The aircraft flew very well, had docile handling qualities, and plenty of power. It handled well on the water (steering and planing) and showed no signs of leakage in the hulls.

\section{Avionics Development}

\section{Flight Control System}

The flight control hardware (Figure 2) used for the ACAT demonstrator is the same system used in MLB's Bat UAV but with software specifically developed for the ACAT airframe and mission. 


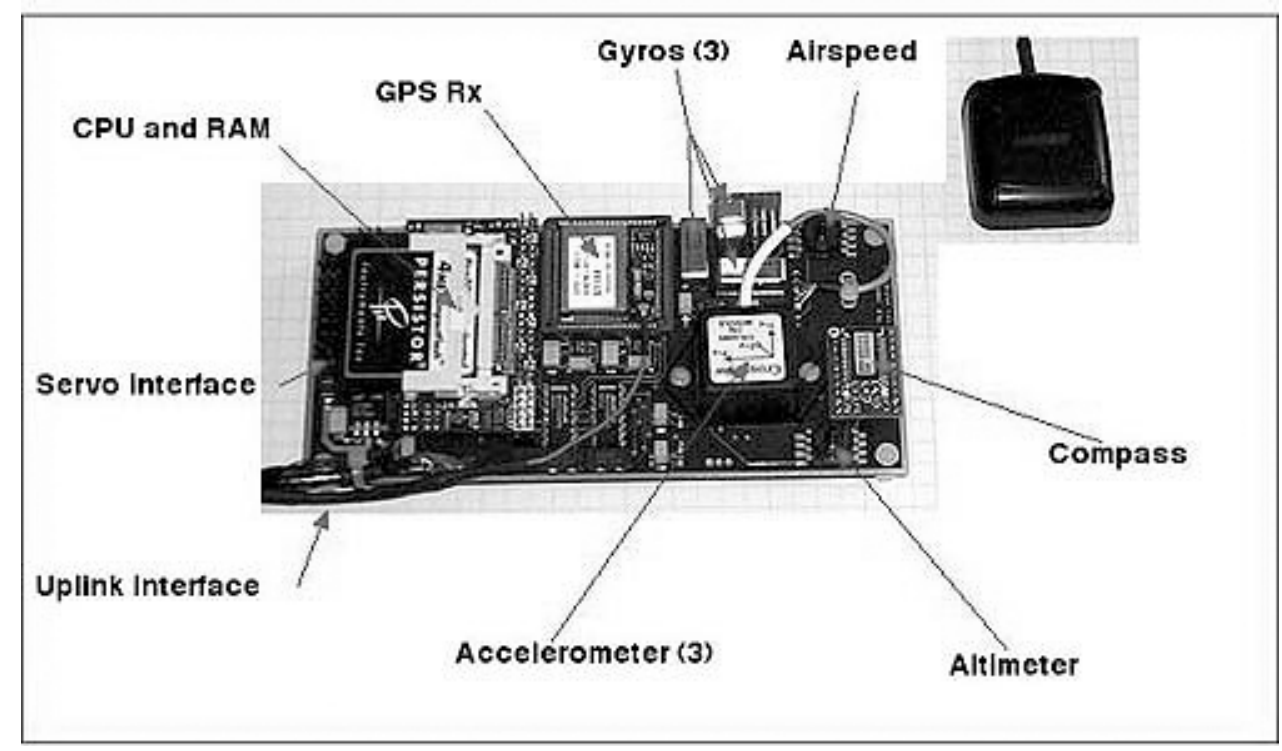

Figure 2: MLB ACAT flight control avionics.

The avionics use automotive grade piezoelectric rate gyros, MEMS based accelerometer, solid-state pressure and altitude sensors, and a miniature 12 channel GPS receiver. These components are mounted to a circuit board that includes a Motorola $68336 \mathrm{CPU}$ programmable in ' $\mathrm{C}$ ', $8 \mathrm{MB}$ flash RAM card for flight data storage, and all interface electronics to the model airplane radio receiver and servos.

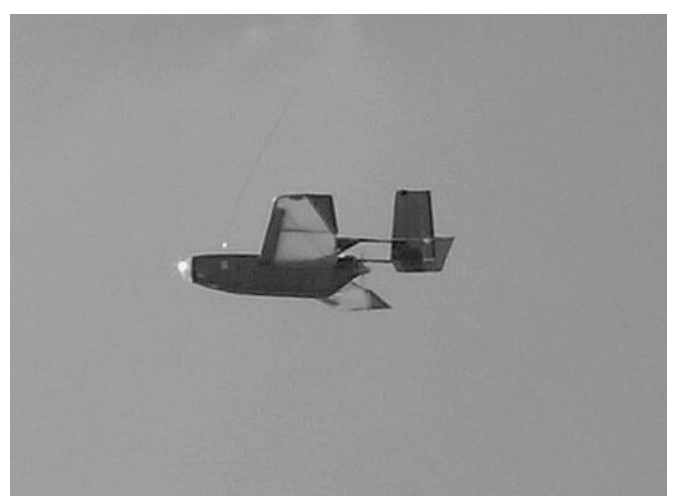

Figure 3. An MLB Bat was used as ACAT flight control avionics test bed.

The hardware was initially tested in an MLB Bat airframe shown in Figure 3. The Bat was flown autonomously between pre-programmed waypoints with manual radio control for launching and landing. A return-to-home capability was developed and tested to insure that the UAV would return to a preprogrammed location in the event that the uplink signal was lost.

\section{MLB Small UAV Ground Station}

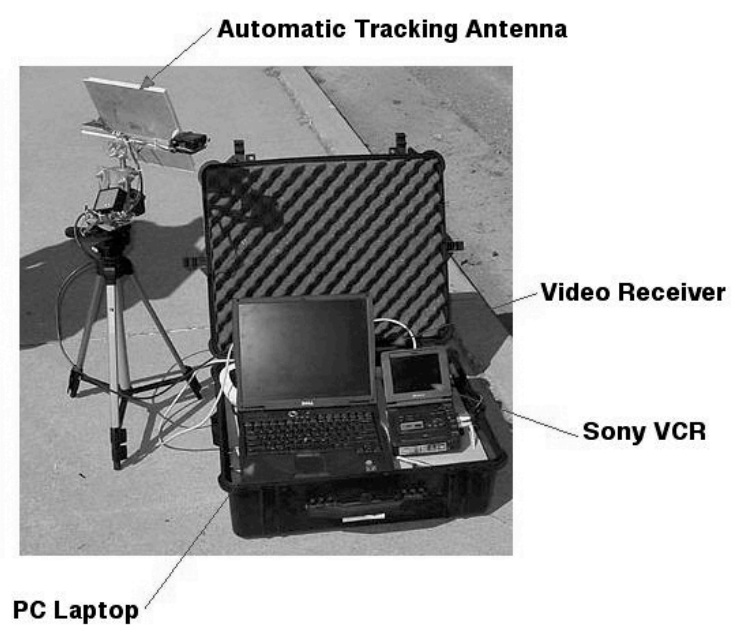

Figure 4. ACAT ground station hardware.

\section{Ground Station}

MLB also developed an integrated ground station that communicates with the flight avionics through a data modem on the video telemetry sound 
channel. The ground station hardware is shown in Figure 4.

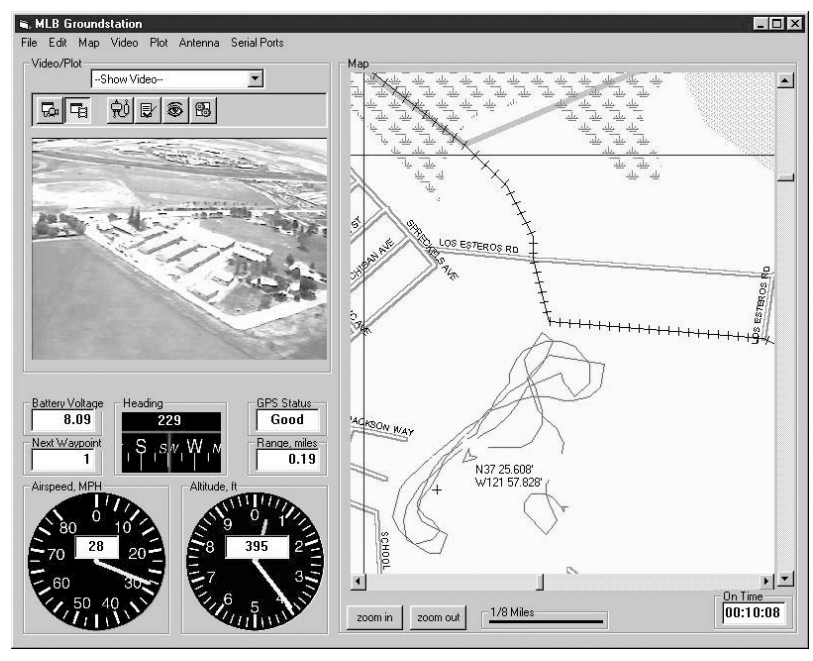

Figure 5. MLB ACAT ground station interface.

The ground station uses a PC laptop as its display console and a screen image of typical flight data is shown in Figure 5.

The ground station shows the aircraft's location on a moving map display that is updated every second. Also displayed are altitude, speed, GPS status, battery voltage, and a choice of flight parameter plots or live video screen. All of the flight data and video is recorded on a ground based Video Cassette Recorder for post-flight analysis.

\section{Integrated Flight Tests}

The MLB avionics were integrated into the ACAT airframe along with the video telemetry downlink system. After installing these components and adjusting the center of gravity between the left and right hulls using lead weight, the ACAT gross weight grew to be two pounds heavier than during radio control flight tests (i.e. $\mathrm{GTOW}=14.2$ pounds). Figure 6 shows the final layout of the equipment in the ACAT aircraft.

Flight tests were conducted from Moffett Federal Airfield at the NASA Ames Research Center. Several sessions were devoted to adjusting the control gains for adequate phugoid and spiral stability and the GPS guidance gains for proper course navigation.

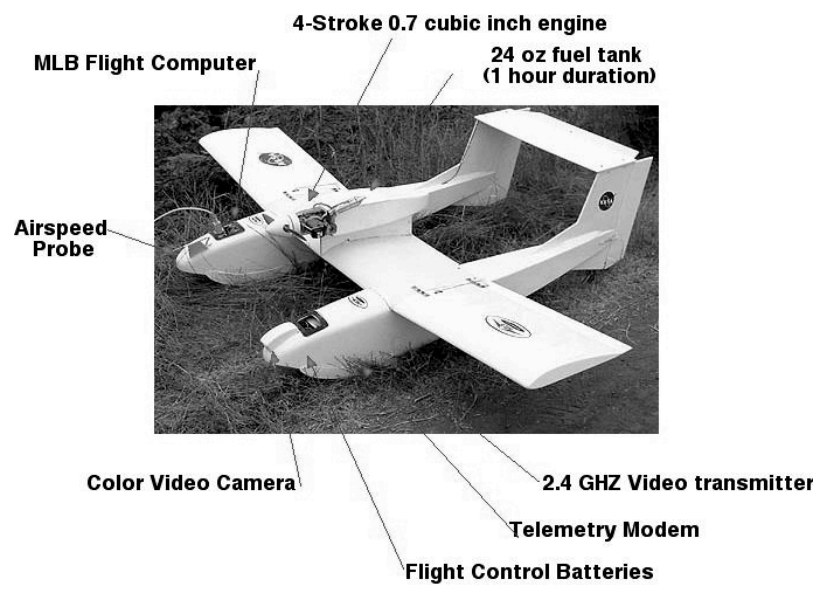

Figure 6. Layout of ACAT hardware within the aircraft.

After many test flights we were able to command airspeed, altitude, and heading with acceptable stability and accuracy. The previously mentioned coupling between engine thrust and pitch moment had made the phugoid motion somewhat oscillatory, but still acceptable. Figures 7 and 8 show the trajectory, altitude, and airspeed during an autonomous flight with navigation between 2 waypoints. The gray portion of the course indicates autonomous flight and the trajectory is slightly oscillatory at times due to a high value for the guidance gain.

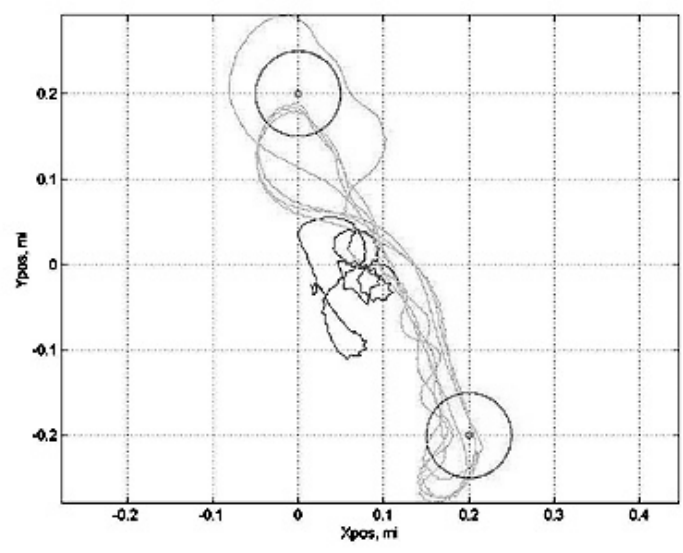

Figure 7. ACAT ground track during autonomous waypoint navigation 

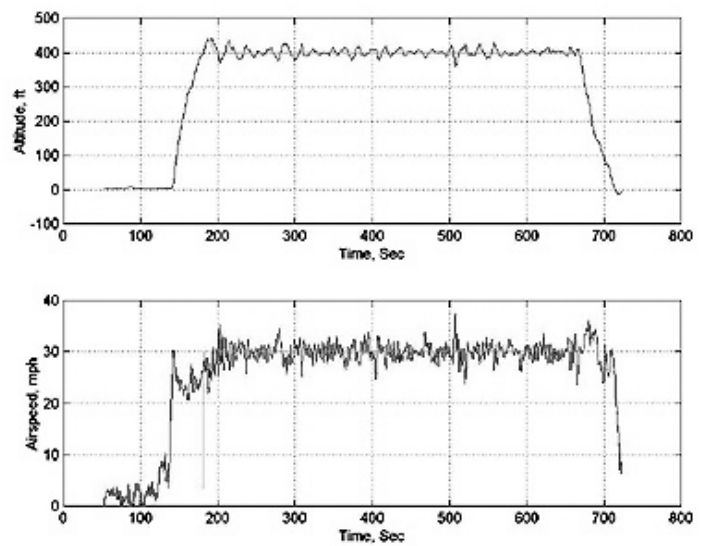

Figure 8. ACAT altitude and airspeed during autonomous waypoint navigation.

\section{Autonomous Take-Off and Landing}

The flight software was updated to include algorithms for autonomous take-off and landing from the Moffett field runway. After several test flights the ACAT accomplished this milestone on July 5, 2001.

The take-off sequence implements yaw rate, roll rate, and pitch attitude feedback below $50 \mathrm{ft}$ altitude and then blends in full control feedback above 50 feet while holding full throttle for takeoff. The sequence is initiated when the autopilot switch is thrown.

The landing sequence requires that waypoints be specified to define a $1 / 2$ mile final approach pattern with 2 waypoints at 0 feet altitude. Below 50 feet the throttle is held at a minimum value and the autopilot flies a constant airspeed $(30 \mathrm{mph})$ approach until touchdown.

Tests of this system were conducted with a reduced GPS guidance gain to cure previously observed heading oscillations. After our fourth flight we were able to successfully perform a complete autonomous flight pattern (take-off, landing pattern, and landing). The only problems noted were:

- $\quad$ The phugoid motion needs more damping. Phugoid oscillations resulted in harder than normal landings causing damage to both fuselages from landing gear loads. We increased the feedback gain from 2.0 to 3.0 to solve this.

- The final descent airspeed (below 20 feet altitude) should be set to $25 \mathrm{mph}$ for slower descent rate at touch down.

- Some heading oscillation was observed prior to touch down and may have due to a slightly too large GPS heading gain.

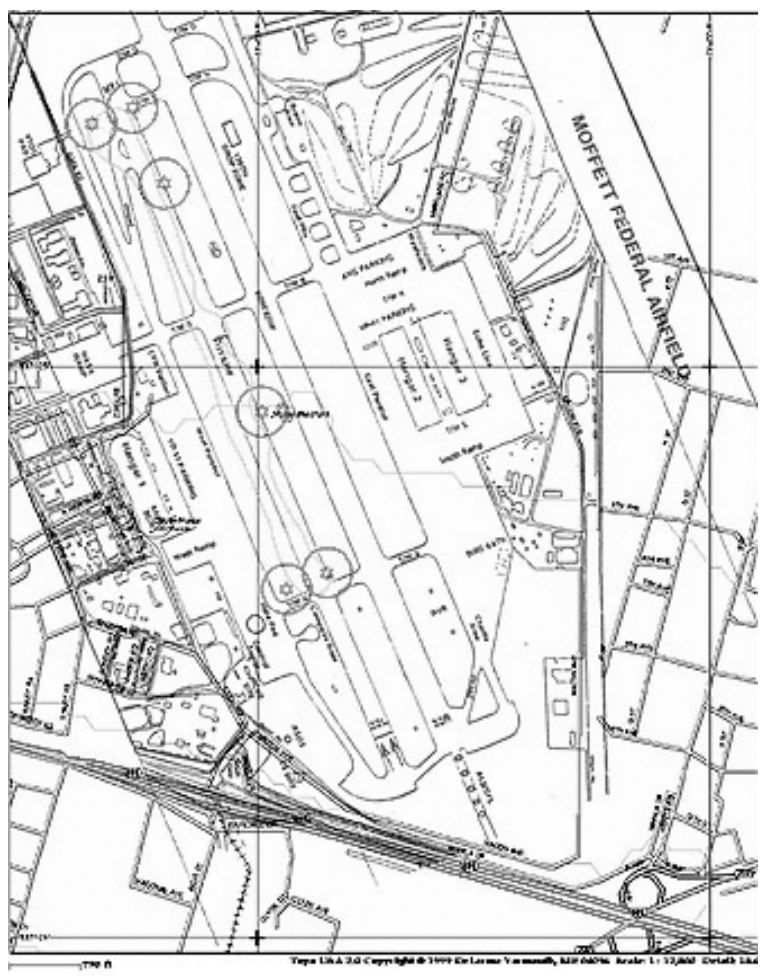

Figure 9. ACAT course during the fully autonomous flight.
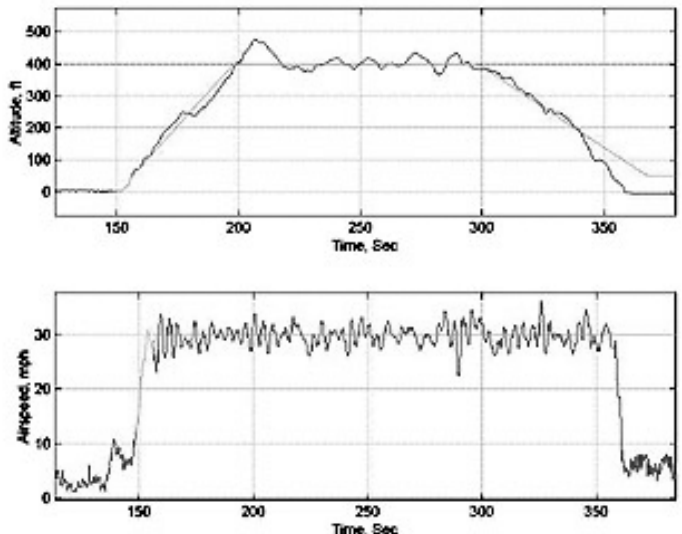

Figure 10. ACAT altitude and speed during fully autonomous flight. 
Figure 9 shows a plot of the course and Figure 10 shows the altitude, and speed for the fully autonomous flight. A map of the Moffett airfield was scanned and superimposed on the data, however, note that it is slightly off-scale and the runway does not exactly line up with the GPS surveyed waypoints.

The plots show the entire flight course in autonomous flight (gray color) except for taxi portions. Take-off and landing occur on runway 32 left between taxiway Alpha and Bravo. There is a large initial altitude overshoot due to integrator wind-up during climb out and this can be eliminated with a feed-forward control on throttle. Note also that the altitude drops below the commanded green line when the terminal approach waypoint is reached and the throttle is cut. This could be improved by using glideslope instead of airspeed control during the final descent.

The aircraft successfully landed on the runway with minor damage caused by overly stiff landing gear and the slight phugoid oscillations.

\section{Autonomous Water Flights}

\section{Initial Flight Attempts}

The final test of the prototype program was to duplicate the autonomous flight from water. Two initial attempts were made and each failed because of poor conditions at the water-flying sites that were available.

The third attempt took place off the Cargill Salt Ponds behind NASA Ames on a windy morning. The wind caused swells that were as deep as the hull height and the ACAT was swamped with salt water during the take-off run. Water from the waves and prop was forced into the hulls through gaps in the wing-hull junction and the access hatches. The combination of waves and hull-spray prevented the ACAT from ever reaching planing speed and the autonomous take-off was aborted. Unfortunately, the salt water damaged the avionics and it was several weeks before repairs were completed. We used this experience to set tight tolerances for wind and wake heights for future operations.

\section{Repair and Reconfirmation}

After the electronics were repaired and the aircraft was more thoroughly waterproofed, a fully autonomous water flight was attempted on September $19^{\text {th }} 2001$. The winds were less than 5 mph and the water surface was smooth. The ACAT performed a successful autonomous water take-off, but began to porpoise severely (phugoid) up and down on climbout. The aircraft achieved waypoints $1 \& 2$ at $400 \mathrm{ft}$ altitude, but the engine then died. The ACAT glided back smoothly $(>1 / 2$ mile with no phugoid oscillations) and landed smoothly on the water.

Subsequent investigations found that the right hull had a small leak near the spray rail allowing a few ounces of water into the bottom of the hull. This was enough to soak a small circuit board that stops RF noise in the elevator servo cable. The board was damaged by the salt water and was replaced. The hull was resealed with epoxy.

We felt that the phugoid instability may have been related to the more rearward CG and the smaller propeller since last flown (the propeller, weight and CG were changed when the aircraft was rebuilt in August).

We decided to re-install fixed Landing Gear and fly from NASA runway to fix the phugoid problem and exercise the engine.

On September $28^{\text {th }}$, three flights off the runways at Moffett Field were performed.

During these flights the longitudinal control gains were adjusted. We found that $\mathrm{Ku}$ de $=-5.0$ $(\mathrm{old}=-10.0)$ and Khdot_dt $=1.0(\mathrm{old}=0.0)$ resulted in good phugoid stability and turn performance. We were then able to fly an autonomous 2-waypoint course with good tracking and altitude-speed hold results.

The engine died while testing the return to home function because of a minor software error (the code for the autonomous landing interfered with the return home function).

With these reconfirmation tests complete, the wheels were removed, the hulls were sealed and we resumed water flying on October 4. 


\section{Successful Flights}

The ACAT UAV demonstrated two fully autonomous flights from water on October 4.

The first attempts at water take-off showed a lot of spray impinging on the propeller at speeds lower than planing speed. The ACAT was able to pass through this regime with slight up elevator trim (to counter the nose down pitch moment from the pylon-mounted engine). Because of crosswinds and that there is no active heading control during takeoff, the ACAT turned 90 degrees during the run and took-off downwind on both flights. This may be correctable in the future by using the compass to steer the plane while on the water.

A rectangular pattern was flown (autonomously) at 400 feet altitude, descending along the final leg to a landing between waypoints 6 $\& 1$ in figure 11. The engine is programmed to stop at 20 feet altitude and the airspeed is reduced to 20 $\mathrm{mph}$ below $50 \mathrm{ft}$ for landing flair. Figure 11 shows the GPS course line for both flights $(1 \& 2)$ and the take-off and landing locations. The course was repeated accurately both times and the ACAT touched down between waypoints $6 \& 1$ as planned. The green portion of the course shows when the ACAT was flying autonomously. The blue portion shows when it was taxiing, being retrieved, or drifting with the wind after landing. The curved flight path of the take-off and then the abrupt correction back onto the course are visible for each of the two flights.

Figures 12 and 13 shows the altitude and airspeed profiles for each flight with the command signal shown in green. Altitude follows the command within \pm 20 feet and airspeed is within \pm 5 mph for most of the flight.

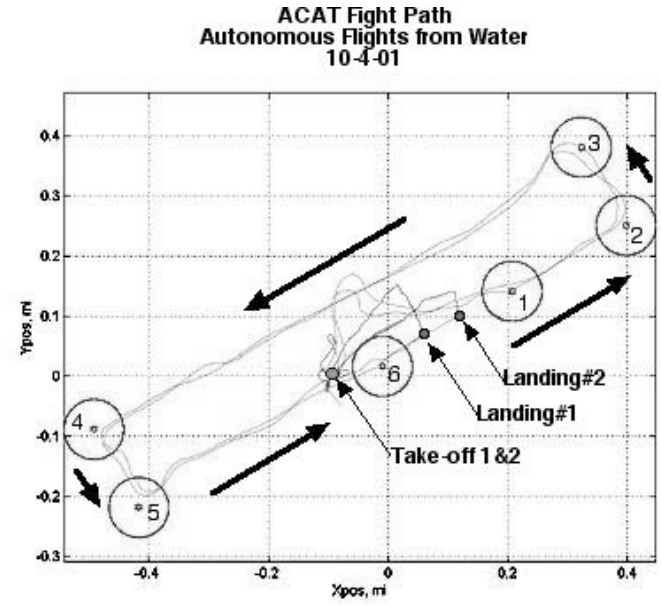

Figure 11: ACAT GPS course for autonomous flights $1 \& 2$.
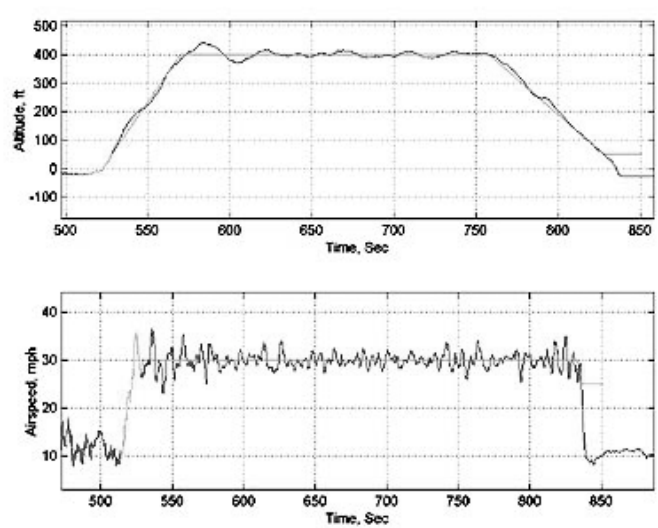

Figure 12: ACAT altitude and speed autonomous flight 1 .
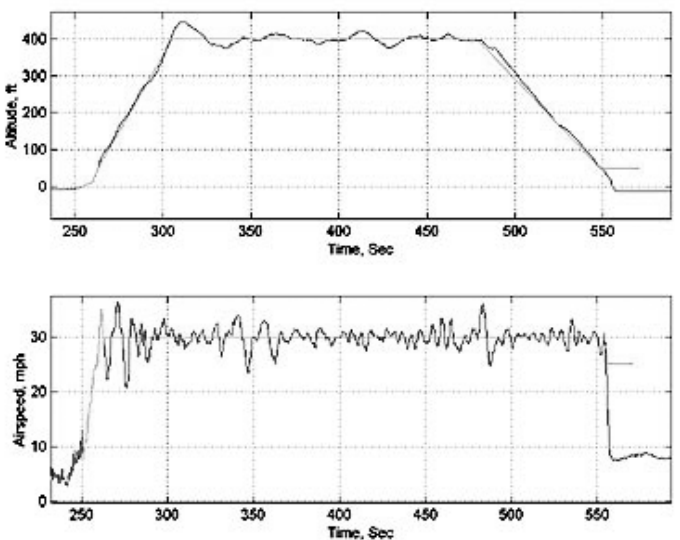

Figure 13: ACAT altitude and autonomous flight 2. 
Figures 15-17 are photographs taken during the final flight tests.

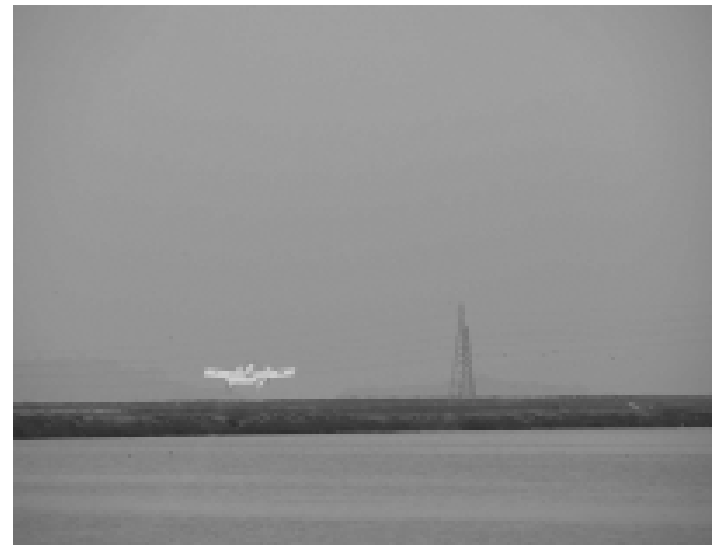

Figure 14. ACAT Autonomous Takeoff

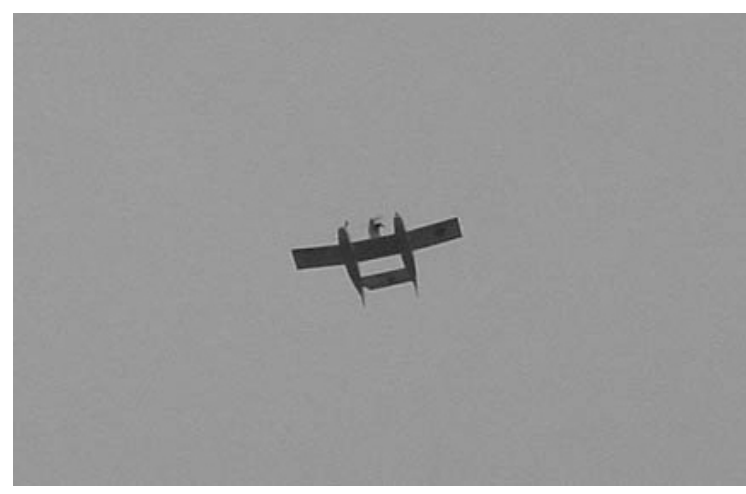

Figure 15. ACAT aircraft in flight.

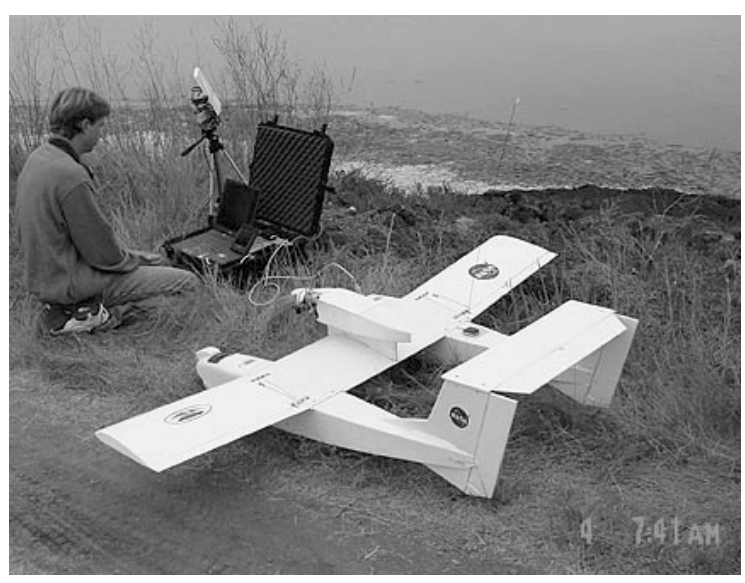

Figure 16. Evaluating flight data and reprogramming the aircraft between flights.

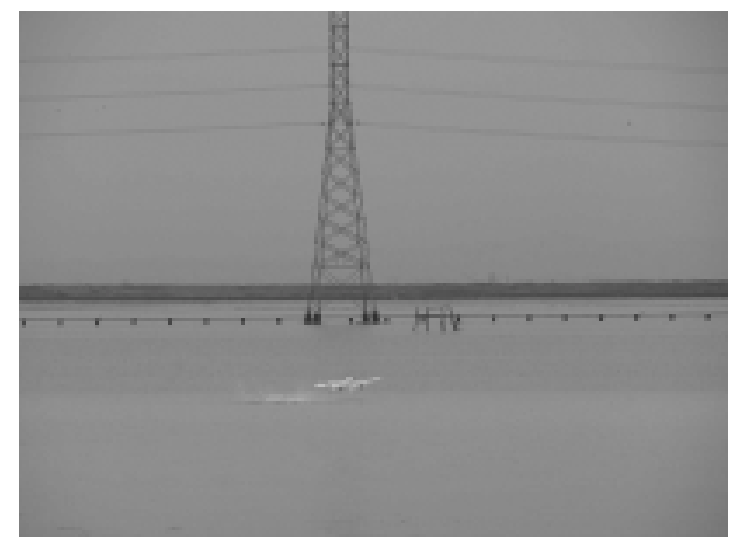

Figure 17. A one-skip landing of the ACAT.

\section{Summary}

The ACAT successfully completed its intended goals, demonstrating autonomous takeoff, waypoint following and landing from both land and water environments. Many things were learned during the development, including salt-water operations, flight planning, and how to work with the NASA flight operations group.

Although the development of the Phase II demonstrator was not pursued, the completion of the prototype has opened up other opportunities for UAV development. Projects such as the BEES (Biologically Inspired Engineering for Exploration Systems) for Mars program and an Intelligent Maneuvering project have given us new missions goals with which to drive development.

Our current goals for development include:

- Incorporating a two-way modem link to allow the aircraft to be controlled from the ground and reprogrammed in flight.

- Integrating a secondary processor on board that would allow new autonomy, system monitoring and diagnosis, and aircraft control algorithms to be evaluated.

\section{Acknowledgements}

This work was funded by Dr. Yuri Gawdiak under the IT Base program at the NASA Ames Research Center. 